NASA

AVSCOM

Technical Memorandum 105718

Technical Report 92-C-003

\title{
Minimization of Deviations of Gear Real Tooth Surfaces Determined by Coordinate Measurements
}

F.L. Litvin, C. Kuan, and J.-C. Wang

University of Illinois

Chicago, Illinois

R.F. Handschuh

Propulsion Directorate

U.S. Army Aviation Systems Command

Lewis Research Center

Cleveland, Ohio

J. Masseth

Dana Corporation

Fort Wayne, Indiana

and

N. Maruyama

Nissan Motor Corporation

1 Natsushima-cho, Yokosuka, Japan

Prepared for the

1992 Power Transmission and Gearing Conference

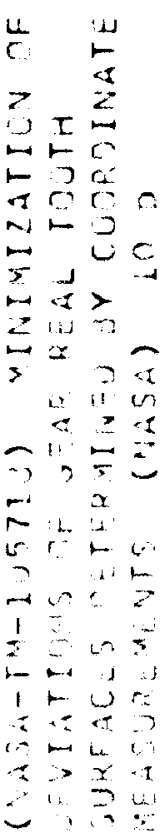

Phoenix, Arizona, September 13-16, 1992

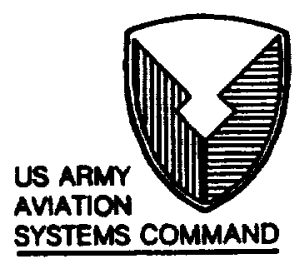





\title{
MINIMIZATION OF DEVIATIONS OF GEAR REAL TOOTH SURFACES DETERMINED BY COORDINATE MEASUREMENTS
}

\author{
F. L. Litvin, C. Kuan and J.-C. Wang \\ (University of Illinois at Chicago) \\ R.F. Handschuh (NASA Lewis Research Center) \\ J. Masseth (the Dana Corporation) \\ N. Maruyama (the Nissan Motor Co.)
}

\begin{abstract}
The deviations of a gear's real tooth surface from the theoretical surface are determined by coordinate measurements at the grid of the surface. A method has been developed to transform the deviations from Cartesian coordinates to those along the normal at the measurement locations. Equations are derived that relate the first order deviations with the adjustment to the manufacturing machine-tool settings. The deviations of the entire surface are minimized. The minimization is achicved by application of the least-square method for an overdetermined system of linear equations. The proposed method is illustrated with a numerical example for hypoid gear and pinion.
\end{abstract}

\section{INTRODUCTION}

Coordinate measurements of gear tooth surfaces coupled with the ability to correct the initially applied machine-tool settings is becoming a significant part of advanced gear technology. We may consider two stages of this technique:

(i) Application of coordinate measurements of the manufactured gears for numerical delermination, in 3D space, of deviations of real tooth surfaces.

(ii) The goal of minimization of deviations can be achieved by proper corrections of initially applied machine-tool settings. The determination of corrected machine-tool settings is found numerically.

The technological aspects of the problem to-be discussed are as follows:

(i) The deviations of real tooth surfaces are inevitable due to surface distortion by heat-treatment, errors of initial machinetool settings, deflection by manufacturing, etc.

(ii) Application of an additional finishing operation for elimination of the deviations would be too expensive in comparison with the approach based on corrections of initially applied machine-tool settings. The advantage of this approach is the possibility of using the same equipment to correct the deviations.
The disadvantage is that the approach will be successful only if the deviations are repeatable.

(iii) The coordinate measurements must be performed with high precision, which currently prohibits them from being performed simultaneously with the manufacturing. Therefore, the coordinate measurements are performed after manufacturing, but only the first gear of the whole gear set to-be manufactured is tested.

(iv) In some cases master-gears are used and the coordinate measurements provide the information about the deviations from the master-surface for the surface being tested. The authors consider this approach less effective as compared to computerized determination of surface deviations and corrections of machinetool settings.

The mathematical solutions to this problem are represented in the Appendix to this paper. The technique described in the paper has been developed in the response to the increasing requirements of high quality gear transmissions. Minimizing the deviations of real tooth surfaces results in a reduction in the level of transmission errors that cause gear noise and vibration.

The proposed approach is applied to hypoid gear drives that have found a wide application in transmissions $[1,2]$. The contents of the paper are complemented with a numerical example for a hypoid pinion and gear to illustrate the effectiveness of the proposed apprnach. The level of deviations of the pinioñ surface has been reduced from 30 microns to the theoretical level of $2-3$ microns.

\section{OVERVIEW OF MEASUREMENT AND MOD. ELLING METHOD}

The approach developed in this paper enables the determination of deviations of a real surface from the known theoretical surface. This is accomplished by two steps: (i) coordinate mcasurements for determination of surface deviations and (ii) minimization of the deviations through correction of the previously applied machine-tool settings. 
The surface deviations obtained initially in Cartesian coordinates are transformed into deviations along the normal to the theoretical surfacc. The coordinate mcasurements are performed by a machine with four or five degrees-of-freedom. In the case of four degrees-of-freedom, the probe performs three translational motions (fig. 1); the fourth motion, rotation, is performed by a rotary table. The axis of rotational motion coincides with the axis of the workpiece. In the case of a five degree-of-freedom machine, the fifth degree of freedom is used to provide the deflections of the probe in the direction of the normal to the thcoretical surface. The probe is provided with a changeable spherical surface whose diameter can be chosen from a wide range.
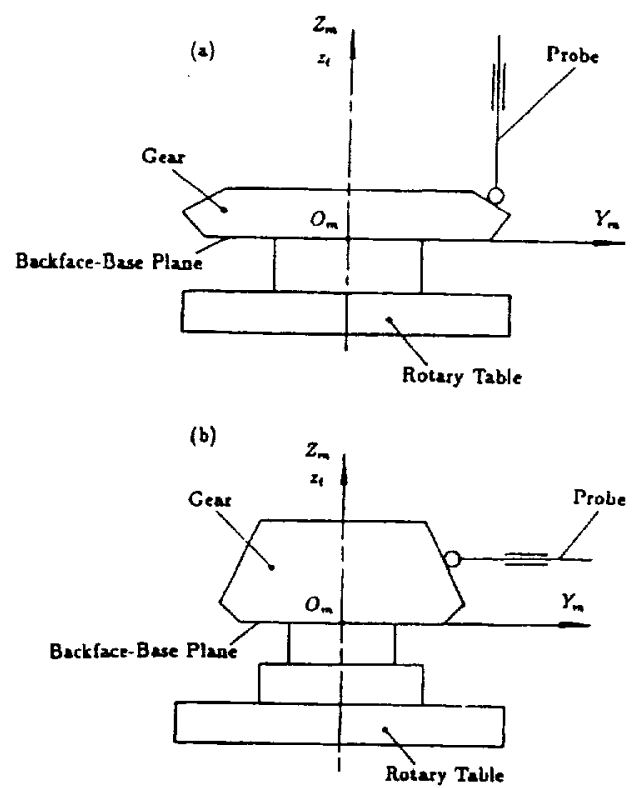

Fig. 1. Surface Measurement.

The motions of the probe and the workpiece by coordinate measurements are computer controlled and therefore a grid comprising of the set of surface points to be measured must be chosen (fig. 2). There is a reference point on the grid that is necessary for the initial installments of the probe. There are two orientations of the probe installment that are used to measure a geat (fig. l(a)) and a pinion (fig. l(b)), depending on the angle of the pitch cone.

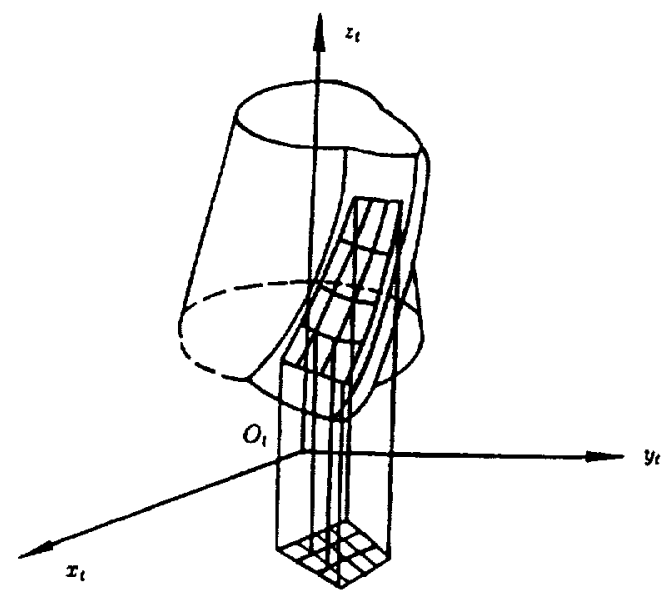

Fig. 2. Grid.
The mathematical aspects of coordinate measurements will now be described [2]: First, it is necessary to derive the equations of the theoretical surface. In many cases this surface can be derived as the envelope to the family of generating surfaces, namely the tool surfaces. Next, the results of coordinate measurements must be transformed into deviations of the real surface represented in the direction of the surface normal. Then, the relations between the surface variations and the corrections to the machine-tool settings must be determined. The surface deviations obtained from coordinate measurements and the surface variations determined by the corrections of machine-tool settings can be represented by an overdetermined system of lincar equations. The number of these equations, $k$, is equal to the number of points of the grid, and the number of unknowns, $m$, is equal to the number of corrections of machine-tool settings $(m \ll k$ ). The optimal solution to such a system of linear equations results in the determination of the machine-tool setting corrections.

\section{EQUATIONS OF THEORETICAL TOOTH SUR- FACE $\Sigma$,}

Considering that the theoretical surface can be determined directly, we represent it in coordinate system $S_{i}$ in two paramctric form as:

$$
\mathbf{r}_{t}(u, \theta), \quad \mathbf{n}_{t}(u, 0)
$$

Here: $\mathbf{r}_{t}$ and $\mathbf{n}_{t}$ are the position-vector and the surface unit normal, respectively; $(u, \theta)$ are the Gaussian coordinates (surfacc coordinates).

For the case when surface $\Sigma_{t}$ is the envelope to the family of generating surface $\Sigma_{c}$, we represent surface $\Sigma_{l}$ and the unit. normal $n_{t}$ to $\Sigma_{t}$ in $S_{t}$ as [3]

$$
\begin{array}{ll}
\mathbf{r}_{t}=\mathbf{M}_{t c} \mathbf{r}_{c}\left(u_{c}, \theta_{c}\right), & f\left(u_{c}, \theta_{c}, \phi\right)=0 \\
\mathbf{n}_{t}=\mathbf{L}_{t c} \mathbf{n}_{c}\left(u_{c}, \theta_{c}\right), & f\left(u_{c}, \theta_{c}, \dot{\phi}\right)=0
\end{array}
$$

Here: $\left(u_{c}, \theta_{c}\right)$ are the Gaussian coordinates of the generating surface $\Sigma_{c} ; \phi$ is the generalized parameter of motion in the process for generation. The equation of meshing is given by:

$$
f\left(u_{c}, \theta_{c}, \phi\right)=N^{(c)} \cdot v^{(c t)}=0
$$

where $N^{(c)}$ is the normal to $\Sigma_{c}, v^{(c t)}$ is the relative motion for a point of contact of $\Sigma_{c}$ and $\Sigma_{t}$. The $4 \times 4$ matrix $\mathbf{M}_{t c}$ and $3 \times 3$ matrix $\mathbf{L}_{t c}$ describe the coordinatc transformation from $S_{c}$ to $S_{t}$ of a position vector and surface unit normal, respectively. Position vectors in 3-D space are represented with homogeneous coordinates.

\section{COORDINATE SYSTEMS USED FOR COORDI- NATE MEASUREMENTS}

Coordinate systems $S_{m}$ and $S_{t}$ arc rigidly connected to the coordinate measuring machine (CMM) and the workpiecc being measured, respectively (fig. 3). The back face of the gear is installed flush with the base plane of the CMM. The distance l between the origins $O_{m}$ and $O_{t}$ is known but the parameter of orientation $\delta$ must be determined (see section t). The coordi- 
nate transformation from $S_{t}$ to $S_{m}$ is represented by the matrix equation

$$
r_{m}=M_{m i} r_{t}
$$

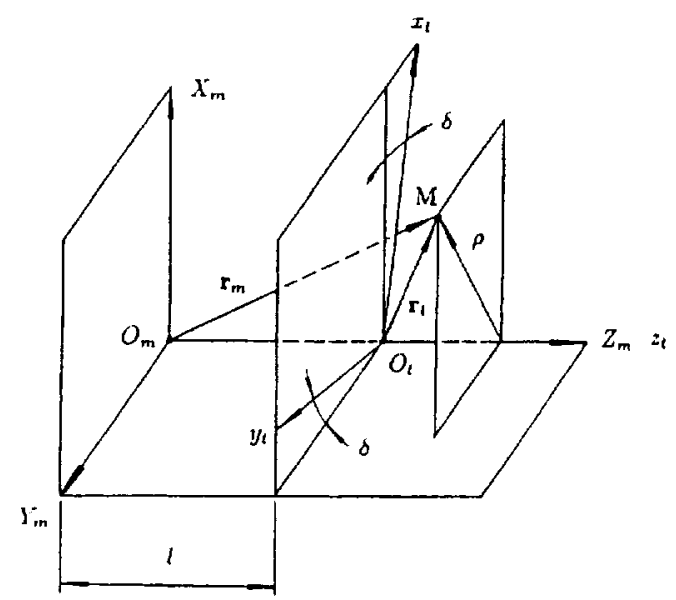

Fig. 3. Coordinate Transformation.

\section{GRID AND REFERENCE POINT}

The grid is a sct of points on $\Sigma_{t}$ chosen as points of contact between the probe and $\Sigma_{t}$ (fig. 3). Fixing the value of $z_{t}$ for the point of the grid, and the valuc of, say $y_{t}$ (or $x_{t}$ ), we can obtain the following equations

$$
y_{t}\left(u_{i}, \theta_{i}\right)=h_{i}, z_{t}\left(u_{i}, \theta_{i}\right)=l_{i}(i=1, \ldots, k)
$$

where $k$ is the number of grid points.

We consider $h_{i}$ and $l_{i}$ as given and solve equations (6) for $\left(u_{i}, \theta_{i}\right)$. Then we can determine the position vectors and the unit normals for $k$ points of the grid using the equations

$$
\begin{gathered}
\mathbf{r}_{t}^{(i)}=\left[\begin{array}{lll}
x_{t}\left(u_{i}, \theta_{i}\right) & y_{t}\left(u_{i}, \theta_{i}\right) & z_{t}\left(u_{i}, \theta_{i}\right)
\end{array}\right]^{r}, \quad(i=1, \ldots, k) \\
\mathbf{n}_{i}^{(i)}=\left[\begin{array}{lll}
n_{x t}\left(u_{i}, \theta_{i}\right) & n_{y t}\left(u_{i}, \theta_{i}\right) & n_{z t}\left(u_{i}, \theta_{i}\right)
\end{array}\right]^{T}, \quad(i=1, \ldots, k)
\end{gathered}
$$

The position vector for the center of the probe, if the deviations are zero, is represented by the equation

$$
\mathbf{R}_{i}^{(i)}=\mathbf{r}_{i}^{(i)}+\rho \mathbf{n}_{i}^{(i)} \quad(i=1, \ldots, k)
$$

where $\rho$ is the radius of the probe tip.

The reference point

$$
\mathbf{r}_{l}^{(0)}=\left[x_{t}\left(u^{(0)}, \theta^{(0)}\right) \quad y_{t}\left(u^{(0)}, \theta^{(0)}\right) \quad z_{t}\left(u^{(0)}, \theta^{(0)}\right)\right]^{T}
$$

is usually chosen as the mean point of the grid.

The center of the probe that corresponds the reference point on $\Sigma_{l}$ is determined from equation (9) as

$$
\mathbf{R}_{t}^{(0)}=\left[X_{t}\left(u^{(0)}, \theta^{(0)}\right) \quad Y_{t}\left(u^{(0)}, \theta^{(0)}\right) \quad Z_{t}\left(u^{(0)}, \theta^{(0)}\right)\right]^{T}
$$

Here: $\left(u^{(0)}, \theta^{(0)}\right)$ are known values.

The coordinates of the reference center of the probe arc represented in coordinate system $S_{m}$ of the measuring machine by the matrix equation

$$
\mathbf{R}_{m}^{(0)}=\mathbf{M}_{m t}(\delta) \mathbf{R}_{l}^{(0)}
$$

Equation (12) yields

$$
\left.\begin{array}{l}
x_{m}^{(0)}=x_{m}^{(0)}\left(\delta, u^{(0)}, \sigma^{(0)}\right) \\
y_{m}^{(0)}=y_{m}^{(0)}\left(\delta, u^{(0)}, \theta^{(0)}\right) \\
z_{m}^{(0)}=z_{m}^{(0)}\left(\delta, u^{(0)}, \theta^{(0)}\right)
\end{array}\right\}
$$

The three equations (13) contain four unknowns: $\delta, x_{m}^{(0)}, y_{m}^{(0)}$ $z_{m}^{(0)}$. To solve these equations we may consider that one of the coordinates of the reference point of the probe center, say $y_{m}^{(0)}$, may be chosen equal to zero. Then the system of equations (13) allows the determination of $\delta, x_{m}^{(0)}$ and $z_{m}^{(0)}$ [2]. Coordinates $x_{m}^{(0)}, y_{m}^{(0)}=0, z_{m}^{(0)}$ are necessary for the initial installment of the center of the probe.

\section{DEVIATIONS OF THE REAL SURFACE}

The deviations of the real surface are caused by errors of manufacturing, heat treatment, ctc. Vector positions of the center of the probe for the theoretical surface and the real surfacc can be represented as follow

$$
\begin{aligned}
& \mathbf{R}_{m}=\mathbf{r}_{m}(u, \theta)+\rho \mathbf{n}_{m}(u, \theta) \\
& \mathbf{R}_{m}^{*}=\mathbf{r}_{m}(u, \theta)+\lambda \mathbf{n}_{m}(u, \theta)
\end{aligned}
$$

Here: $\mathbf{r}_{m}$ and $\mathbf{n}_{m}$ are the position vector and the unit normal to the theoretical surface, respectively, that are represented in coordinate systcm $S_{m}$ of the measuring machine; $\lambda$ determincs the real location of the probe center and is considered along the normal to the theoretical surface; $\mathbf{R}_{m}$ and $\mathbf{R}_{m}^{*}$ represent in $S_{n}$ the position vector of the probe center for the theoretical and real surfaces, respectively. Equations (14) and (15) yield

$$
\mathbf{R}_{m}^{*}-\mathbf{R}_{m}=(\lambda-\rho) \mathbf{n}_{m}=\Delta n \mathbf{n}_{m}
$$

and

$$
\Delta n=\left(\mathbf{R}_{m}^{*}-\mathbf{R}_{m}\right) \cdot \mathbf{n}_{m}
$$

The position vector $\mathbf{R}_{m}^{*}$ is determined by coordinate measurements for points of the grid. Equation (17) determines numerically the function:

$$
\Delta n_{i}=\Delta n_{i}\left(u_{i}, \theta_{i}\right) \quad(i=1, \ldots, k)
$$

that represents the deviations of the real surface for each point of the grid.

\section{MINIMIZATION OF DEVIATIONS}

The procedure of minimization of deviations can be repre- 
sented in two stages: (i) detcrmination of variations of theoretical surface caused by changes of applied machine-tool settings, and (ii) minimization of deviations of real surface by appropriate correction of machine-tool scttings.

We consider that the theoretical surface is represented in $S_{t}$ as

$$
r_{t}=r_{i}\left(u, \theta, d_{j}\right) \quad(j=1, \ldots, m)
$$

where parameters $d$, are the machine-tool settings.

The surface variations are represented by

$$
\delta \mathbf{r}_{t}=\frac{\partial \mathbf{r}_{t}}{\partial u} \delta u+\frac{\partial \mathbf{r}_{t}}{\partial \theta} \delta \theta+\sum_{j=1}^{n} \frac{\partial \mathbf{r}_{t}}{\partial d_{j}} \delta d_{j}
$$

We multiply both sides of equation (20) by the surface unit normal $\mathbf{n}_{\mathbf{t}}$ and take into account that $\frac{\partial \mathbf{r}_{t}}{\partial \theta} \cdot \mathbf{n}_{\mathrm{t}}=\frac{\partial \mathbf{r}_{t}}{\partial u} \cdot \mathbf{n}_{t}=0$ since $\frac{\partial \mathbf{r}_{t}}{\partial \theta}$ and $\frac{\partial \mathbf{r}_{t}}{\partial u}$ lie in the plane that is tangent to the surface. Then
we obtain:

$$
\delta \mathbf{r}_{t} \cdot \mathbf{n}_{t}=\left(\sum_{j=1}^{m} \frac{\partial \mathbf{r}_{t}}{\partial d_{j}} \cdot \mathbf{n}_{t}\right) \delta d_{j}=\sum_{j=1}^{m} \mathbf{a} \delta d_{j}
$$

We can now consider a system of $k$ linear equations in $m$ unknowns ( $m \ll k$ ) of the following structure

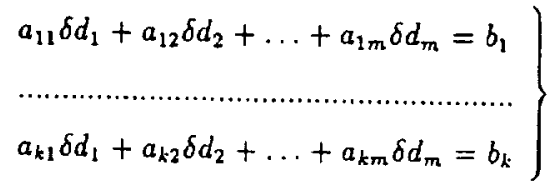

Here:

$$
b_{i}=\Delta n_{i}=\left(\mathbf{R}_{m i}^{*}-\mathbf{R}_{m i}\right) \cdot \mathbf{n}_{m i}
$$

where $i$ designates the number of grid point; $a_{g j}(s=1, \ldots, k$; $j=1, \ldots, m)$ represent the dot product of partial derivatives $\frac{\partial \mathbf{r}_{\mathbf{t}}}{\partial d_{i}}$ and unit normal $\mathbf{n}_{\mathbf{t}}$.

The system of linear equations (22) is overdetermined since $m \ll k$. The essence of the procedure of minimization of deviations is determination of such unknowns $\delta d_{j}(j=1, \ldots, m)$ that will minimize the difference between the left and right sides of equations (22). The solution was accomplished by the leastsquare method. The subroutine DLSQRR of IMSL MATH / LIBRARY [4] was used for computerization of the procedure.

The success of minimization of deviations depends on the number of parameters that may be varied (the number of machine-tool settings that may be corrected). The number of pinion machine-tool settings is larger than for the gear. The minimization of deviations can be performed for each pinion tooth side separately. However, it must be performed simultaneously for both sides of the gear tooth since the gear is cut by the duplex method. For these reasons the minimization of deviations for the pinion is more effective than for the gear (see below the numerical examples).

\section{APPLICATION TO INSPECTION OF FORMATE HYPOID GEAR}

Each tooth side of a formate face-milled gear is generated by

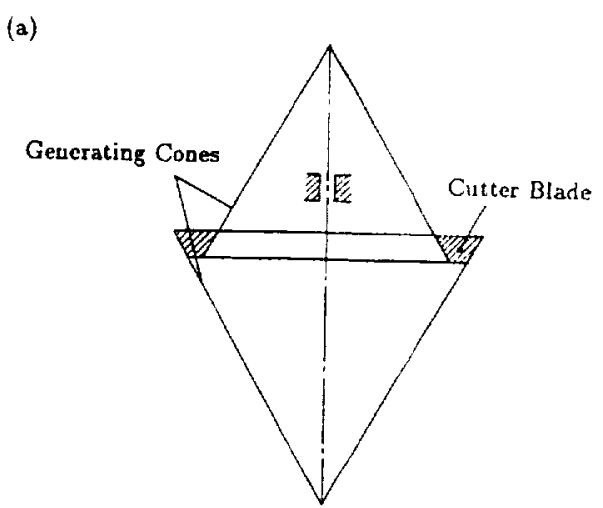

(b)

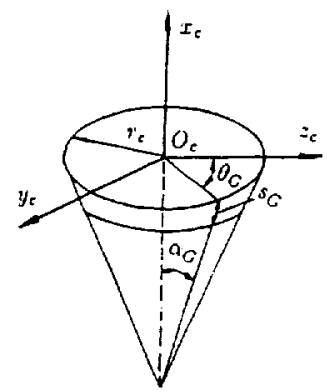

Fig. 4. Generating Cones for Format Face-milled Gear.

a cone and the gear tooth surface is the surface of the generating cone. Two cones that are shown in fig. 4(a) represent both sides of the gear space. The following equations represent in coordinate system $S_{c}$ gear surfaces for both sides and the unit normals to such surfaces (fig. 4(b))

$$
\begin{gathered}
\mathbf{r}_{c}=\left[\begin{array}{c}
-s_{G} \cos \alpha_{G} \\
\left(r_{c}-s_{G} \sin \alpha_{G}\right) \sin \theta_{C} \\
\left(r_{c}-s_{G} \sin \alpha_{G}\right) \cos \theta_{G} \\
1
\end{array}\right] \\
\mathbf{n}_{c}=\left[\begin{array}{c}
\sin \alpha_{C} \\
-\cos \alpha_{G} \sin \theta_{C} \\
-\cos \alpha_{G} \cos \theta_{G}
\end{array}\right]
\end{gathered}
$$

Here: $\boldsymbol{r}_{c}$ is the position vector and $\mathbf{n}_{c}$ is the surface unit normal; $r_{c}$ is the cutter tip radius; $\alpha_{G}$ is the cutter blade angle $\left(\alpha_{G}>0\right.$ for the concave side and $\alpha_{G}<0$ for the convex side).

Fig. 5 shows the installment of the generating cone on the cutting machine. Coordinate systems $S_{0}$ and $S_{t}$ are rigidly connected to the cutting machine and the gear being generated, respectively. Systems $S_{c}, S_{o}$ and $S_{t}$ are rigidly connected to eacl other since the gear is formate cut (no relative motion between the cutter and workpiece). To represent in $S_{l}$ the theoretical gear tooth surface $\Sigma_{t}$ and the unit normal to $\Sigma_{t}$ we use the following matrix equations

$$
\boldsymbol{r}_{t}\left(s_{G}, \theta_{G}, d_{j}\right)=\mathbf{M}_{t c} \mathbf{r}_{c}\left(s_{G}, \theta_{G}\right)
$$




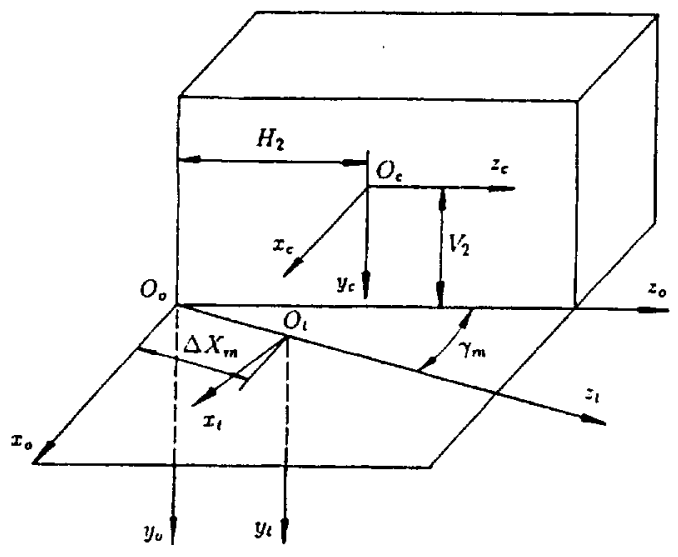

Fig. 5. Machine-tool Settings for Formate Face-milled Gear.

$$
\mathbf{n}_{\imath}\left(s_{G}, O_{G}, d_{j}\right)=\mathbf{L}_{\imath c} \mathbf{n}_{c}\left(s_{G}, \theta_{G}\right)
$$

wherc

$$
\begin{aligned}
M_{t c} & =M_{i o} M_{o c} \\
& =\left[\begin{array}{cccc}
\cos \gamma_{m} & 0 & -\sin \gamma_{m} & 0 \\
0 & 1 & 0 & 0 \\
\sin \gamma_{m} & 0 & \cos \gamma_{m} & -\Delta X_{m} \\
0 & 0 & 0 & 1
\end{array}\right]\left[\begin{array}{cccc}
1 & 0 & 0 & 0 \\
0 & 1 & 0 & -V_{2} \\
0 & 0 & 1 & H_{2} \\
0 & 0 & 0 & 1
\end{array}\right]
\end{aligned}
$$

The surface Gaussian coordinates are $s_{G}$ and $\theta_{G}$ and $d_{j}\left(\gamma_{m}\right.$, $V_{2}, H_{2}$ and $\left.\Delta X_{m}\right)$ are the machine-tool settings.

The numerical example presented in this paper is based on the experiment that has been performed at the Dana Corporation (Fort Wayne, USA). The initial deviations $\Delta n$ for each side of real tooth surface have been obtained by measurements on a coordinate measuring machine (fig. 1). The grid for the measurements is formed by nine sections along the tooth length with each section having five points. The number $k$ of grid points is therefore 45 and the reference point is at the middle of the grid, (i.e., the third point of the fifth section). In the measurement, the coordinate $y_{m}^{(0)}$ of the reference point is chosen to be zcro and the alignment angle $\delta$ is determined from solving equation system (13).

The minimization of deviations was performed in accordance to the algorithm described in scction 6 for the formate cut gear. The measurement of the inilial and final deviations are shown in figures 6-8. The machine-tool settings initially used and corrected are shown in Table 1.

\section{EQUATIONS OF PINION THEORETICAL TOOTH SURFACE}

The pinion tooth surface is generated as the envelopc to the family of tool surfaces that are cone surfaces (fig. 9).

Henceforth, we will consider the following coordinate systems:

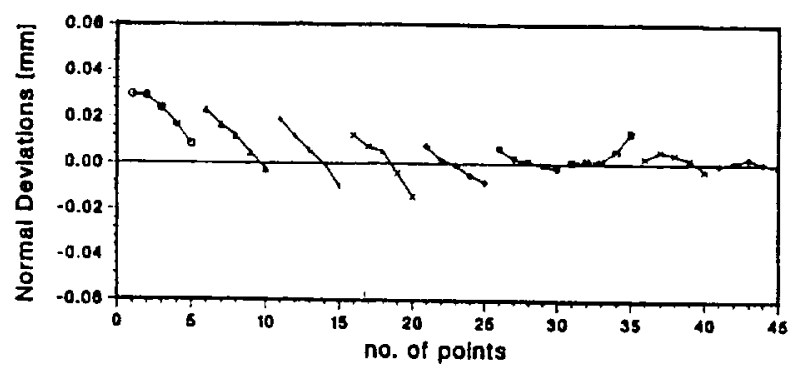

Fig. 6. Deviations of Gear Real Tooth Surface (Driving Side).

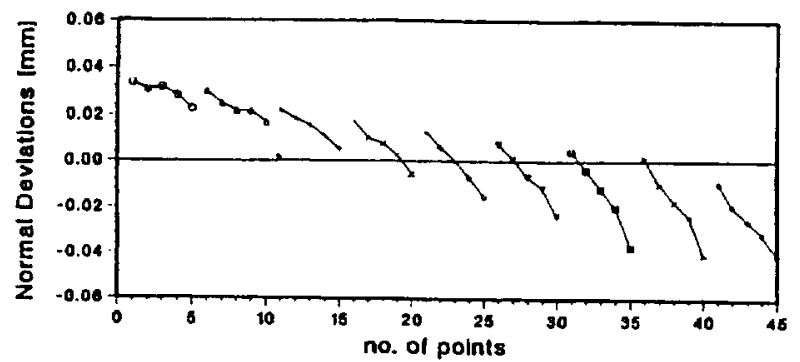

Fig. 7. Deviations of Gear Real Tooth Surface (Coast Side).

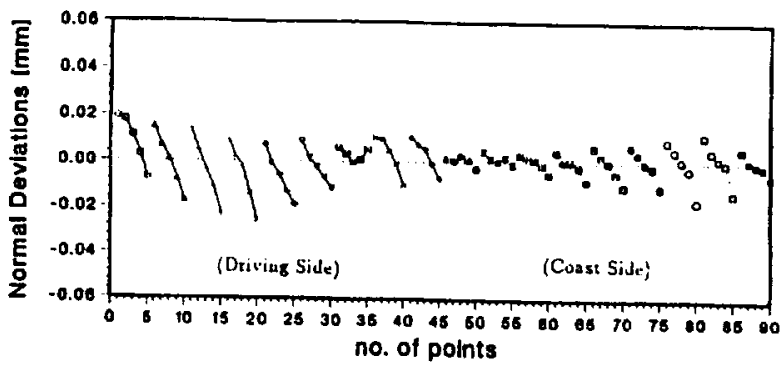

Fig. 8. Minimized Deviations of the Gear.

Table 1: Results of Gear Minimization

\begin{tabular}{|l|c|c|}
\hline Machine Setting & Initial & Corrected \\
\hline Pressure Angle $\alpha_{G}$ & $21.25^{\circ}$ & $21.25^{\circ}$ \\
Cutter Dismeter, mm (in) & $228.6(9)$ & $228.6(9)$ \\
Point Width of Cutter, mm (in) & $2.03(.08)$ & $2.03(.08)$ \\
$V_{2}, \mathrm{~mm}$ (in) & $103.252550(4.06506)$ & $103.25220(4.06505)$ \\
$H_{2}, \mathrm{~mm}$ (in) & $27.4666(1.08136)$ & $27.21603(1.07150)$ \\
$\gamma_{m}, \mathrm{rad}$. & 1.059816 & 1.00437 \\
$\Delta X_{m}, \mathrm{~mm}$ (in) & $0.009677(.00038)$ & $-0.53343(-0.0210)$ \\
\hline
\end{tabular}

(i) the fixed ones, $S_{0}\left(x_{0}, y_{0}, z_{0}\right)$ and $S_{q}\left(x_{q}, y_{q}, z_{q}\right)$ that are rigidly connected to the cutting machine (fig. 10 and fig. 11); (ii) the movable coordinate systems $S_{c}$ and $S_{p}$ that are rigidly connected to the cradle of cutting machine and the pinion, respectively; (iii) coordinate system $S_{t}$ that is rigidly connected to the head cutter. In the process of generation the cradle with $S_{c}$ perlorms rotational motion about the $z_{0}$-axis with angular velocity $\omega^{(c)}$, and the pinion with $S_{p}$ performs rotational motion about the $x_{q}$-axis with angular velocity $\omega^{(p)}$ (fig. 11).

The tool (head-cutter) is mounted on the cradle and performs rotational motion with the cradle. Coordinate system $S_{t}$ is rigidly connected to the cradle. To describe the installment of the tool 


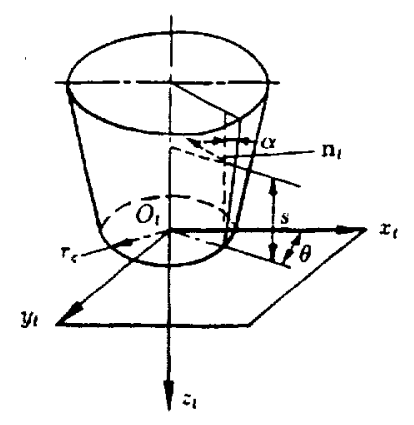

(a) Head-Cutter Surface Paramcters

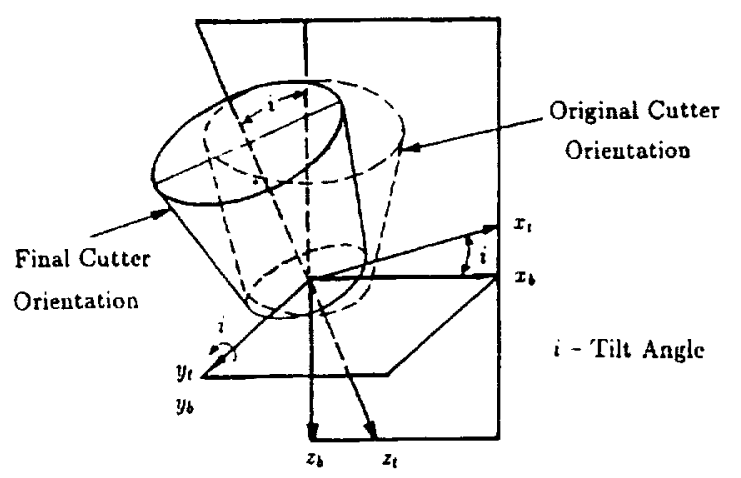

(b) Coordinate Systens for Head-Cutter Tilt

Fig. 9. Pinion Head-cutter Surface.

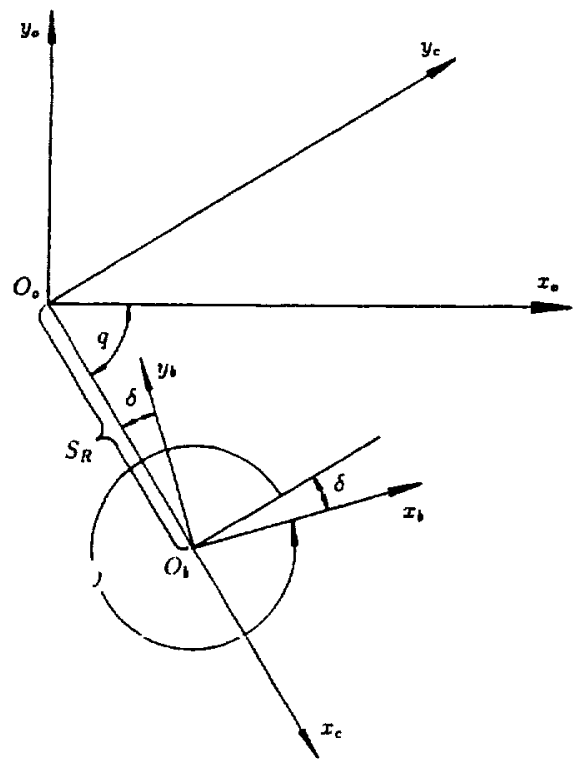

Fig. 10. Cutting Machine and Cradle Coordinate Systems.

with respect to the cradle we use coordinate system $S_{b}$ (fig. 9 and fig. 10). The requircd orientation of the head-cutter with respect to the cradle is accomplished as follows: (i) coordinate systems $S_{b}$ and $S_{l}$ are rigidly connected and then they are turned as one rigid body about the $z_{c}$-axis through the swivel angle $j=2 \pi-\delta$ (fig. 10); (ii) then the head-cutter with coordinatc system $S_{t}$ is tilted about the $y_{b}$-axis under the angle $i$ (fig. $9(\mathrm{~b})$ ). The headcutter is rotated about its axis $z_{t}$ but the angular velocity in this

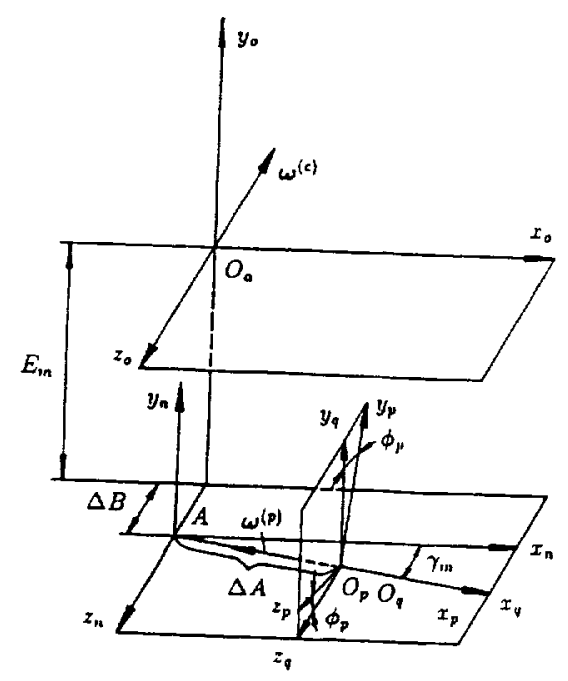

Fig. 11. Angular Velocities of Cradle and Pinion.

motion is not related with the gencration process and depends only on the desired velocity of cutting.

It will be shown later that the deviations of real pinion tootlt surface can be minimized by corrections of parameters of installment of the pinion and the head-cutter. These pinion setting parameters are $E_{m}$ - the machine offset, $\gamma_{m}$ - the machine-root angle, $\Delta B$ - the sliding base, $\triangle A$ - the machine center to back (fig. 11). The head-cutter settings parameters are: $S_{R^{-}}$radial setting, $O_{c}$ - initial value of cradle angle, $j$ - the swivel angle (fig. 10), and $i$ - the tilt angle (fig. $9(\mathrm{~b})$ ).

\section{Tool Surface Equations}

The head-cutter surface is a cone and is represented in $S_{t}$ (fig. 9) as

$$
\mathbf{r}_{t}(s, \theta)=\left[\begin{array}{c}
\left(r_{c}+s \sin \alpha\right) \cos \theta \\
\left(r_{c}+s \sin \alpha\right) \sin \theta \\
-s \cos \alpha \\
1
\end{array}\right]
$$

Here: $(s, \theta)$ are the Gaussian coordinates, $\alpha$ is the blade angle and $r_{c}$ is the cutter point radius. Vector function (29) with $a$ positive and $\alpha$ negative represents surfares of two head-cuttcrs that are used to cut the pinion concave side and convex sidc, respectively.

The unit normal to the head-cutter surface is represented in $S_{t}$ by the equations

$$
n_{1}=\left[\begin{array}{lll}
-\cos \alpha \cos \theta & -\cos \alpha \sin \theta & -\sin \alpha
\end{array}\right]^{T}
$$

The Family of Tool Surfaces is represented in $S_{p}$ by the matrix equation

$$
\mathbf{r}_{p}\left(s, \theta, \phi_{p}\right)=\mathbf{M}_{p q} \mathbf{M}_{q n} \mathbf{M}_{n o} \mathbf{M}_{o c} \mathbf{M}_{c b} \mathbf{M}_{b t} \mathbf{r}_{t}(s, \theta)
$$

Here: $S_{n}$ is an auxiliary fixed coordinate system whose axcs parallel to $S_{0}$ axes. 


$$
\begin{aligned}
& \mathbf{M}_{b t}=\left[\begin{array}{cccc}
\cos i & 0 & \sin i & 0 \\
0 & 1 & 0 & 0 \\
-\sin i & 0 & \cos i & 0 \\
0 & 0 & 0 & 1
\end{array}\right] \\
& \mathbf{M}_{c b}=\left[\begin{array}{cccc}
-\sin j & -\cos j & 0 & S_{R} \\
\cos j & -\sin j & 0 & 0 \\
0 & 0 & 1 & 0 \\
0 & 0 & 0 & 1
\end{array}\right] \\
& \mathbf{M}_{o c}=\left[\begin{array}{cccc}
\cos q & \sin q & 0 & 0 \\
-\sin q & \cos q & 0 & 0 \\
0 & 0 & 1 & 0 \\
0 & 0 & 0 & 1
\end{array}\right] \\
& \mathbf{M}_{n o}=\left[\begin{array}{cccc}
1 & 0 & 0 & 0 \\
0 & 1 & 0 & E_{m} \\
0 & 0 & 1 & -\Delta B \\
0 & 0 & 0 & 1
\end{array}\right] \\
& \mathbf{M}_{q n}=\left[\begin{array}{cccc}
\cos \gamma_{m} & 0 & \sin \gamma_{m} & -\triangle A \\
0 & 1 & 0 & 0 \\
-\sin \gamma_{m} & 0 & \cos \gamma_{m} & 0 \\
0 & 0 & 0 & 1
\end{array}\right] \\
& \mathbf{M}_{p q}=\left[\begin{array}{cccc}
1 & 0 & 0 & 0 \\
0 & \cos \phi_{p} & \sin \phi_{p} & 0 \\
0 & -\sin \phi_{p} & \cos \phi_{p} & 0 \\
0 & 0 & 0 & 1
\end{array}\right]
\end{aligned}
$$

$\delta=2 \pi-j_{i} q=\theta_{c}+m_{c p} \phi_{p}$ where $\theta_{c}$ is the initial cradle angle and $m_{c p}=\omega^{(c)} / \omega^{(p)}$.

\section{Equation of Meshing}

This equation is represented as [3]:

$$
\mathbf{n}^{(p)} \cdot \mathbf{v}^{(c p)}=\mathbf{N}^{(p)} \cdot \mathbf{v}^{(c p)}=f\left(s, \theta, \phi_{p}\right)=0
$$

where $n^{(p)}$ and $N^{(p)}$ are the unit normal and the normal to the tool surface, and $v^{(c p)}$ is the velocity in relative motion.

Equation (32) is invariant with respect to the coordinate system where the vectors of the scalar product are represented. These vectors in our derivations have been represented in $S_{o}$ as follows,

$$
\begin{gathered}
\mathbf{n}_{o}=\mathbf{L}_{o c} \mathbf{L}_{c b} \mathbf{L}_{b t} \mathbf{n}_{t} \\
\mathbf{v}_{o}^{(c p)}=\left[\left(\omega_{o}^{(c)}-\omega_{o}^{(p)}\right) \times \mathrm{r}_{a}\right]+\left(\overline{O_{0} A} \times \omega_{o}^{(p)}\right)
\end{gathered}
$$

Here:

$$
\begin{aligned}
& \mathbf{r}_{o}=\mathbf{M}_{o c} \mathbf{M}_{c b} \mathbf{M}_{b t} \mathbf{r}_{\mathrm{t}} \\
& \overline{O_{o} A}=\left[\left.\begin{array}{lll}
0 & -E_{\mathrm{m}} & \triangle B
\end{array}\right|^{T}\right. \\
& \boldsymbol{\omega}_{o}^{(p)}=-\left[\left.\begin{array}{lll}
\cos \gamma & 0 & \sin \gamma
\end{array}\right|^{T} ; \quad\left(\left|\omega_{0}^{(p)}\right|=1\right)\right. \\
& \omega_{o}^{(c)}=-\left[\begin{array}{lll}
0 & 0 & m_{c p}
\end{array}\right]^{T}
\end{aligned}
$$

\section{Pinion Tooth Surface}

Equations (31) and (32) represent the pinion tooth surface in three-parametric form with parameters $s, \theta$ and $\phi_{p}$. However, since equation (32) is linear with respect to $s$ we can eliminate $s$ and represent the pinion tooth surface in two-parametric form as

$$
\mathbf{r}_{p}\left(\theta, \phi_{p}, d_{j}\right)
$$

Here: $d_{j}(j=1, \ldots, 8)$ designate the installment paramcters: $E_{\mathrm{m}}, \gamma_{\mathrm{m}}, \triangle B, \triangle A_{1} S_{R}, 0_{c}, j$ and $i$.

The normal to the pinion tooth surface is represented as

$$
\mathrm{n}_{p}\left(\theta, \phi_{p}, d_{k}\right)
$$

where $d_{k} \quad(k=1,2,3,4)$ designate the installment parameters $\gamma_{m}, \theta_{c}, j$ and $i$.

\section{Results of Minimization}

Fig. 12 and fig. 13 illustrate the initial deviations $\Delta b_{i}$ of the real surface, that have been obtained by measurements and calculations for the concave side and convex side, respectively. The blank data, the basic machine-tools settings, the corrections of machine-tool settings and the corrected machine-tool settings are shown in Table 2-3. Based on the corrected machine-tool settings, we can manufacture a new surface that will optimally fit the theoretical surface after the surface is distorted by manufacturing processes and heat treatment. The minimized deviations between the new surface and the theoretical surface are shown in fig. 14 and fig. 15. These figures confirm the effectiveness of the proposed approach. The deviations of approximately 30 microns have been reduced to $2-3$ microns.

\section{CONCLUSION}

A general approach for computcrized determination of devi ations of a real surface from the theoretical one based on coordinate measurements has been proposed. An algorithm for mini. mization of deviations by corrections of initially applied machinetool settings through application of a least square approach has been developed. The approach is illustrated with an example of the tooth surface of a hypoid pinion and gear.

\section{ACKNOWLEDGEMENT}

This research has received financial support from the NASALewis Research Center, Gleason Memorial Fund, the Dana Corporation and the Nissan Motor Co.

\section{REFERENCES}


1. Gleason Works, 1987, "G-Age T.M. User's Manual ", for the Gleason Automated Gear Evaluation System Used with Zeiss Coordinate Measuring Machines.

2. Litvin, F.L., Zhang, Y., Kieffer, J., Handschuh, R. F., "Identification and Minimization of Deviations of Real Gear Tooth Surfaces", ASME Journal of Mechanical Design, Mar. 1991, pp. 55-62.

3. Litvin, F. L., 1989, "Theory of Gearing", NASA publications; RP-1212 (AVSCOM technical report; 88-c-035).

4. Dongarra, J. J., Bunch, J. R., Moler, C. B., and Stewart, G. W., 1979, "LINPACK User's Guide", SIAM, Philadelphia.

5. Litvin, F. L., Zhang, Y., Kuan, C., and Handschuh, R. F., "Computerized Inspection of Real Surfaces and Minimization of their Deviations", Proceedings of International Conference on Metrology and Properties of Engincering Surfaces, England, 10th - 12th, April 1991.

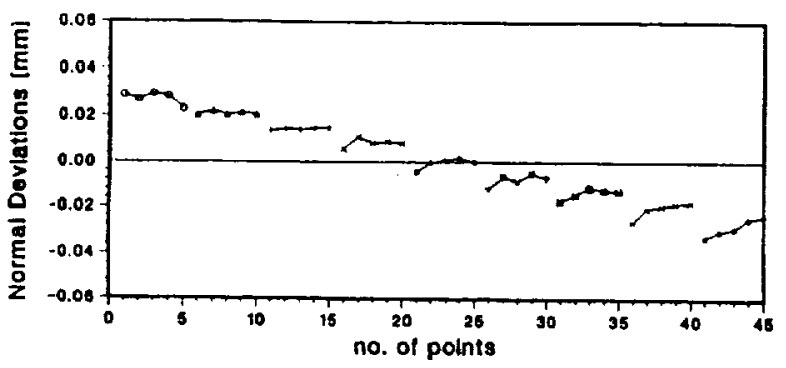

Fig. 12. Deviations of Pinion Real Tooth Surface (Concave Side).

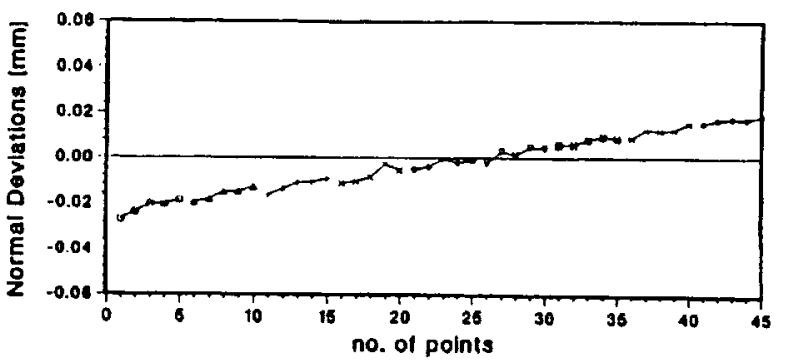

Fig. 13. Deviations of Pinion Real Tooth Surface (Convex Side).
Table 2: Blank Data of Hypoid Pinion

Number of Teeth $=13$
Shaft Angle $=1.57079 \mathrm{rad}$.
Pitch Diameter $=88.22 \mathrm{~mm}$
Outside Diameter $=103.96 \mathrm{~mm}$
Pitch Angle $=0.32055 \mathrm{rad}$.
Face Anglc $=0.41480 \mathrm{rad}$.
Root Angle $=0.30136 \mathrm{rad}$.
Mean Spiral Angle $=0.84677 \mathrm{rad}$.
Face Width $=38.30 \mathrm{~mm}$
Whole Width $=11.63 \mathrm{~mm}$
Hand of Spiral $:$ R. H.

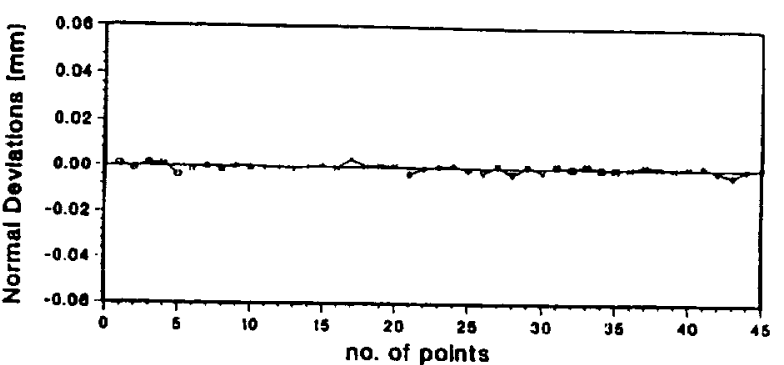

Fig. 14. Minimized Deviations of the Pinion (Concave Side).

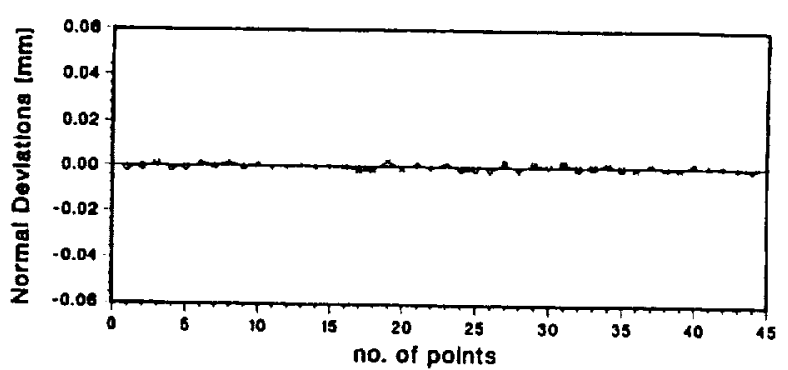

Fig. 15. Minimized Deviations of the Pinion (Convex Side).

Table 3: Basic, Currecled, and Machine-Tool Selling Differences of the Pinion

(Unit: Length in mm; Angle in rad.)

\begin{tabular}{|c|c|c|c|c|c|c|}
\hline \multirow[t]{2}{*}{ Machine Setting } & \multicolumn{2}{|c|}{ Bavic Machine-Tool Settings } & \multicolumn{2}{|c|}{ Corrected Machine-Tool Settings } & \multicolumn{2}{|c|}{ Setting Diferences } \\
\hline & Convex Side & Concave Side & Convex Side & Concave Side & Convex Sido & Concave Side \\
\hline Bacic Tilk Angle & 0.3761899 & 0.4104054 & 0.3712125 & 0.4360375 & $.0 .1977365 \mathrm{E}-02$ & $0.2563208 \mathrm{E}-01$ \\
\hline Swivel Angle & 5.766247 & 6.000656 & 5.768892 & 6.042021 & $0.2644968 \mathrm{E}-02$ & $0.4136530 \mathrm{E}-01$ \\
\hline Machine Rool Angle & 6.233736 & 6.229372 & 6.236861 & 6.202894 & $0.3125239 \mathrm{E}-02$ & $-0.2647790 \mathrm{E}-01$ \\
\hline Cradle Angle & 4.846199 & 1.566173 & 4.845308 & 1.573228 & $-0.8908187 \mathrm{E}-03$ & $0.7054806 \mathrm{E}-02$ \\
\hline Radiej Setting & 114.0236 & 109.6660 & 113.6455 & 110.4463 & -0.3780939 & 0.7803197 \\
\hline Sliding Bace & 23.87000 & 14.82000 & 23.87000 & 14.82000 & $0.0000000 E+\infty$ & $0.0000000 E+\infty 0$ \\
\hline Mechine Center to Back & 3.280000 & -3.100000 & 3.767510 & -3.970493 & $0.5769074 \mathrm{E}-01$ & -0.5540259 \\
\hline Blank Oftset & -40.12000 & -34.58000 & -39.63248 & -35.45049 & 0.4875103 & -0.8704924 \\
\hline Cutting Ratio & 0.3020416 & 0.3230215 & 0.3020446 & 0.3230215 & $0.0000000 E+00$ & $0.0000000 E+\infty$ \\
\hline Cutter Point Radius & 114.9350 & 113.0300 & 114.9350 & 113.0300 & $0.0000000 \mathrm{E}+00$ & $0.0000000 \mathrm{E}+\infty$ \\
\hline Cutter Blade Angle & -0.5410521 & 0.2443461 & -0.5410521 & 0.2443461 & $0.0000000 E+\infty 0$ & $0.0000000 \mathrm{E}+\infty$ \\
\hline
\end{tabular}


$\longrightarrow$ 
Public reporting burden for this collection of information is estimated to average 1 hour per response, including the time for reviewing instructions, searching existing data sources. gathering and maintaining the data needed, and completing and reviewing the coilection of information. Send comments regarding this burden estimate or any other aspect of this collection of intormation, inctuding suggestions for reducing this burden, to Washington Headquarters Services, Directorate tor intormation Operations and Reports. 1215 Jefferson Davis Highway. Suite 1204, Arlington, VA 22202-4302, and to the Office of Management and Budget, Papenwork fleduction Project (0704-0188). Washington, DC 20503.

\begin{tabular}{|l|l|l|l|}
\hline 1. AGENCY USE ONLY (Leave blank) & $\begin{array}{r}\text { 2. REPORT DATE } \\
\text { September } 1992\end{array}$ & $\begin{array}{r}\text { 3. REPORT TYPE AND DATES COVERED } \\
\text { Technical Memorandum }\end{array}$ \\
\hline
\end{tabular}

\section{TITLE AND SUBTITLE}

Minimization of Deviations of Gear Real Tooth Surfaces Determined

by Coordinate Measurements

\section{AUTHOR(S)}

F.L. Litvin, C. Kuan, J.-C. Wang, R.F. Handschuh,

WU-505-63-36

J. Masseth, and N. Maruyama

7. PERforming organization NAME(S) AND ADDRESS(ES)

NASA Lewis Research Center

Cleveland, Ohio 44135-3191

and

Propulsion Directorate

U.S. Army Aviation Systems Command

Cleveland, Ohio 44135-3191

9. SPONSORING/MONITORING AGENCY NAMES(S) AND ADDRESS(ES)

National Acronautics and Space Administration

Wushington, D.C. 20546-10001

and

U.S. Army Aviation Systems Command

St. Louis, Mo. 63120-1798
5. FUNDING NUMBERS

11. SUPPLEMENTARY NOTES

Prepared for the 1992 Power Transmission and Gearing Conference, Phocnix, Arizona, September 13-16, 1992. F.L. Litvin, C. Kuan, and J.-C.

Wang, University of Illinois, Chicago, Illinois 60680. R.F. Handschuh, Propulsion Directorate, U.S. Army Aviation Systems Command. J. Masseth,

Dana Corporation, P.O. Box 750, Fort Wayne, Indiana 46825. N. Maruyama, Nissan Motor Corporation, 1, Natsushima-cho, Yokosuka, 237 Japan. Responsibla person, R.F. Handschuh, (216) 433-3969.

12a. DISTRIBUTION/AVAILABILITY STATEMENT

Unclassified - Unlimited

Subject Category 37

\section{ABSTRACT (Maximum 200 words)}

The deviations of a gear's real tooth surface from the theoretical surface are determined by coordinate measurements at the grid of the surface. A method has been developed to transform the deviations from Cartesian coordinates to those along the normal at the measurement locations. Equations are derived that relate the first order deviations with the adjustment to the manufacturing machine-tool settings. The deviations of the entire surface are minimized. The minimization is achieved by application of the least-square method for an overdetermined system of linear equations. The proposed method is illustrated with a numerical example for hypoid gear and pinion.

14. SUEJECT TERMS

Gear; Grid surface; Deviations of gear; Measurements

15. NUMBER OF PAGES

17. SECURTY CLASSIFICATION OF AEPORT

Unclassified
18. SECUATYY CLASSIFICATION OF THIS PAGE Unclassified
19. SECURIY CLASSIFICATION OF ABSTRACT

Unclassified 
National Aeronautics and

Space Administration

Lewis Reseaveh Conter

Cleveland, Ohio 44135

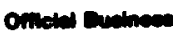

Peneity for Prwase Ves seso
FOUATH CLASS MALL

ADDRESS CORRECTION REQUESTED
|||||

tage and Fees $P$ ard

National Aeronaulics and

Space Administration

NASA 451 
